\title{
Unusual Component Dynamics in Poly(ethylene oxide)/Poly(methyl methacrylate) Blends As Probed by Deuterium NMR
}

\author{
K. L. Ngai and C. M. Roland* \\ Naval Research Laboratory, Washington, D.C. 20375-5342 \\ Received November 26, 2003; Revised Manuscript Received February 11, 2004
}

\begin{abstract}
We analyze recent deuterium NMR data on blends of poly(ethylene oxide) (PEO) with poly(methyl methacrylate) (PMMA), using a model for the blend dynamics based on the coupling model. This model has previously been shown to describe the relaxation properties of blends whose component dynamics are strongly composition dependent. We show herein that the unusual feature of PEO/PMMA blends, an invariance of the PEO dynamics to composition, is a natural consequence of the high frequency and temperature of the NMR experiments. To wit, when the independent relaxation time of our model approaches the characteristic time for intermol ecular cooperativity ( $\sim 2 \mathrm{ps})$, the effect of local envi ronment on the component dynamics becomes negl igible. A similar situation pertains for poly(vinyl methyl ether) mixed with polystyrene, at temperatures sufficiently high that the segmental relaxation times become small. On the other hand, for blends of 1,4-polyisoprene with poly(vinylethylene), the component dynamics retain their dependence on composition, even at high temperatures. The reason for the differing behavior is brought out by an analysis using our blend model.
\end{abstract}

\section{Introduction}

In any research area, continued experimental investigation is the preferred method to advance our understanding as well as to validate theoretical efforts. In particular, when different theories purport to explain the same phenomena, new experimental facts are crucial. The recent deuterium NMR study of blends of deuterated poly(ethylene oxide) ( $\left.\mathrm{d}_{4} \mathrm{PEO}\right)$ and poly(methyl methacrylate) (PMMA) by Lutz et al. ${ }^{1}$ is such an example in the field of component dynamics of miscible polymer blends. The authors found that the segmental dynamics of $d_{4}$ PEO are nearly independent of composition for blends ranging from $0.5 \%$ to $30 \% \mathrm{~d}_{4}$ PEO, over the temperature range studied, and are about 12 orders of magnitude faster than the dynamics of PMMA for a $3 \% \mathrm{~d}_{4} \mathrm{PEO}$ blend near its glass transition temperature, $T_{g}$. Such composition-independent dynamics are unusual in comparison to other miscible blends. Even the $0.5 \% \mathrm{~d}_{4} \mathrm{PEO}$ mixture exhibited identical dynamics to that of 3,6 , and $10 \% \mathrm{~d}_{4} \mathrm{PEO}$. The critical overlap concentration, $\mathrm{c}^{*}$, of $\mathrm{d}_{4} \mathrm{PEO}$ was estimated to be about $1.5 \%$. Certainly the $0.5 \%$ results and possibly also the $3 \% \mathrm{~d}_{4} \mathrm{PEO}$ results can be interpreted as each $\mathrm{d}_{4}$ PEO chain being completely surrounded by PMMA segments. This would eliminate composition fluctuations as a source of the fast $d_{4} P E O$ dynamics and render any blend theory based primarily on composition fluctuation as inapplicable herein. From their results, including for blends at higher $\mathrm{d}_{4} \mathrm{PEO}$ concentrations, in which composition fluctuations become important, Lutz et al. ${ }^{1}$ concluded that the $\mathrm{d}_{4} \mathrm{PEO}$ segmental dynamics for blends from $0.5 \%$ to $30 \% \mathrm{~d}_{4}$ PEO cannot be described by several available models. However, we have developed a model for blend dynamics, ${ }^{2-7}$ which uses the coupling model ${ }^{8}$ to describe the component segmental dynamics while introducing concentration fluctuations and compositional heterogeneity unique to mixtures. The purpose of the present work was to apply our model, since it is applicable to the $d_{4} P E O$ dynamics both in blends with a significant concentration of $\mathrm{d}_{4} \mathrm{PEO}$ and in the dilute $\mathrm{d}_{4}$ PEO limit. In this paper, we show that the predictions of our blend model are consistent with the nearly composition-independent $d_{4} P E O$ segmental dynamics over the entire composition range studied by Lutz et al.

\section{Blend Model Based on the Coupling Model of Relaxation}

One of the first models of the dynamics of polymer blends ${ }^{2-4}$ was based on the intrinsic mobility differences of the components and on the local compositional heterogeneity due to concentration fluctuations. The dynamics of the various relaxing species are determined not only intrinsically, by the chemical composition of each component, but also by the local environment, since the latter governs the intermolecular coupling (cooperativity) associated with the relaxation. Thus, the dynamics of a given species reflects its intrinsic mobility and the degree of constraint imposed by its local environment. The approach adopted by Roland and $\mathrm{Ngai}^{2-4}$ was to use the coupling model, which has previously been applied to describe local segmental relaxation in neat polymers, ${ }^{9-14}$ generalized to describe the relaxation function and the temperature dependence of the relaxation times of the components in binary polymer blends. The correlation function for a neat amorphous polymer has the stretched exponential form

$$
\phi(\mathrm{t})=\phi_{0} \exp \left(-[\mathrm{t} / \tau]^{1-\mathrm{n}}\right)
$$

The observed local segmental relaxation time, $\tau$, depends nonlinearly on the strength of intermolecular coupling, quantified by the magnitude of the parameter n

$$
\tau=\left(\mathrm{t}_{\mathrm{c}}^{-\mathrm{n}} \tau_{0}\right)^{1 / 1-\mathrm{n}}
$$

The relaxation time in the absence of intermolecular coupling (i.e., for $\mathrm{n}=0$ ) is $\tau_{0}$ (the independent, or noncooperative, relaxation time), while $t_{c}$ defines a characteristic time for the onset of the coupling. For polymers, $\mathrm{t}_{\mathrm{c}}$ is about $2 \mathrm{ps} .{ }^{15,16}$ 
Since the coupling parameter depends on chemical structure, ${ }^{9,10}$ the components of a blend have different intermolecular coupling even when in the same blend. A difference in intrinsic mobilities for the components is an implicit feature of an interpretation of blend dynamics based on the coupling model. The components also experience a distribution of local environments due to composition fluctuations, and consequently the relaxation of each will not be equivalently perturbed by neighboring segments. In the language of the coupling model, this means that each component will be associated with a distribution of $\mathrm{n}$. From eq 2, it follows that $\tau$ will also be distributed. Two characteristic features of blend dynamics-thermorheological complexity and reversal in the asymmetry of the dispersion-follow directly from the distribution of $\mathrm{n}$ and eq 2 of our model.

Formally, relaxation of component $i(i=1,2)$ reflects a sum over relaxation in its different local environments

$$
\begin{aligned}
\phi_{\mathrm{i}}(\mathrm{t})=\phi_{\mathrm{i}, 0} \int_{\mathrm{n}_{\mathrm{i}, \text { min }}}^{\mathrm{n}_{\mathrm{i} \text { max }}} \exp \left[-\mathrm{a}_{\mathrm{i}}\left(\mathrm{n}-\hat{\mathrm{n}}_{\mathrm{i}}\right)^{2}\right] \times & \\
& \quad \exp \left\{-\left[\mathrm{t} / \tau_{\mathrm{i}}(\mathrm{n})\right]^{\left.1-\mathrm{n}_{3}\right\} d \mathrm{n}}\right.
\end{aligned}
$$

where $\hat{n}_{i}, n_{i, \max }$, and $n_{i, \min }$ are respectively the most probable, maximum, and minimum coupling parameters of the distribution, the latter reflecting the bounds on the degree of intermol ecular constraints experienced in the heterogeneous environment. (As written, eq 3 assumes the macroscopic response equals the sum of the local responses.) For dielectric relaxation, this linear summation may be appropriate. Alternatively, one could write a similar expression assuming additivity of the displacements. This problem has been treated in detail el sewhere. ${ }^{17} \tau_{\mathrm{i}}(\mathrm{n})$ for any $\mathrm{n}$ is determined by

$$
\tau_{\mathrm{i}}(\mathrm{n})=\left[\mathrm{t}_{\mathrm{c}}^{-\mathrm{n}} \tau_{\mathrm{i}, 0}\right]^{1 /(1-\mathrm{n})}
$$

which is the equivalent of eq 2 for a neat polymer, with the important distinction that the independent relaxation time of the respective components, $\tau_{i, 0}$, will differ. Equation 3 assumes that concentration fluctuations produce a normally distributed range of values of the coupling parameters for each component. An analysis of diel ectric data using this approach has been carried out for blends of poly(vinyl methyl ether)/polystyrene, ${ }^{4}$ tetramethyl polycarbonate/polystyrene, ${ }^{7}$ 1,4-polyisoprene/ poly(vinylethylene), ${ }^{2}$ and polychloroprene/epoxidized polyisoprene. ${ }^{18}$ The model predicts many of the wellknown anomalies seen in miscible blends, such as broad glass transitions, the reversal in asymmetry of the segmental relaxation function, and the breakdown of time-temperature superpositioning, the latter even in the dynamics of one component. The model is applicable not only to binary polymer blends but also to solutions of polymers with molecular liquids. ${ }^{19-24}$ Analyses of the latter have served to explain certain anomalous effects that the addition of polymer can have on the small molecule dynamics, and vice versa. Especially, we note that the model offers verifiable predictions concerning the dynamics of the small molecule component in the dilute limit (i.e., probe molecules). ${ }^{25}$

\section{PEO Dynamics in $\mathbf{d}_{\mathbf{4}}$ PEO/PMMA Blends}

Since we are primarily concerned with the $d_{4} P E O$ dynamics, $i$ in eqs 3 and 4 would denote PEO herein; for convenience, however, we drop the suffix i for all

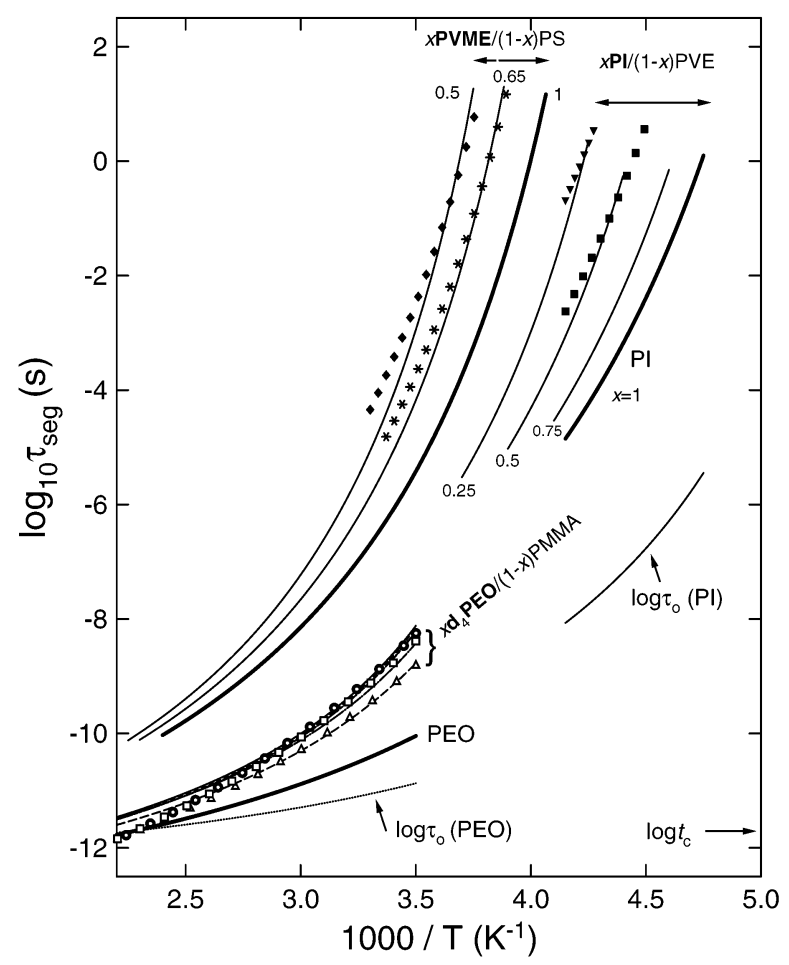

Figure 1. Segmental relaxation times for PEO neat (bold solid line) and in blends with PMMA (dashed lines) containing 3\%$30 \%$ PEO (from top to bottom) taken from the VFTH fits of Lutz et al. ${ }^{1}$ The most probable relaxation times are also shown, as calculated from eq 5 for the lower $(O, \hat{n}=0.76)$, midrange $(\square, \hat{n}=0.75)$, and higher concentrations of PEO $(8, \hat{n}=0.715)$. Also shown is the independent relaxation time for PEO (dotted line, using $n=0.5)$, which lies close to the characteristic time, $\mathrm{t}_{\mathrm{c}}=2 \mathrm{ps}$. Similar results are shown for PI neat (bold solid line) and in blends with PVE containing 25\%-75\% PI (dashed lines), together with the calculated $\tau_{\text {seg }}$ for $25 \%(\nabla)$ and $50 \%$ (ם) compositions. Note the independent relaxation time for $\mathrm{PI}$ (dotted line) is 4 or more decades longer than $t_{c}$. Similar results are included for PVME neat (bold solid line) and blended with PS (thinner lines), along with the calculated curves for $55 \%$ $(\diamond)$ and $65 \%\left(^{*}\right)$ PVME compositions. F or PVME, $\tau_{0}$ (not shown because it overlaps the PEO data) is al so much larger than $t_{c}$ in the temperature range where we calculate $\tau_{\text {seg. }}$.

quantities hereafter. Lutz et al. ${ }^{1}$ fit the deuterium spinlattice relaxation data to a modified KohlrauschWilliams-Watts (KWW) function, from which they deduced the segmental relaxation time, $\tau_{\text {seg, }}$ and the KWW stretch exponent, $\beta$. The temperature depend-

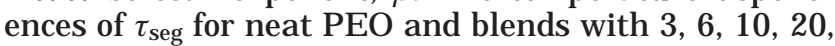
and $30 \% \mathrm{~d}_{4} \mathrm{PEO}$ were presented in terms of the Vogel Fulcher-Tammann-Hesse (VFTH) equation. These results are reproduced in Figure 1, showing the near independence of $\tau_{\text {seg }}$ on composition for blends from $3 \%$ to $30 \% \mathrm{~d}_{4}$ PEO. Lutz et al. ${ }^{1}$ also reported, without showing the data, that the $0.5 \% \mathrm{~d}_{4}$ PEO blend had $\tau_{\text {seg }}$ equivalent to that for the 3,6 , and $10 \%$ compositions.

The application of our model to the $\mathrm{d}_{4}$ PEO dynamics begins with consideration of neat PEO. Substituting $\tau_{\text {seg }}$ of neat PEO for $\tau$ in eq 2, the independent relaxation time $\tau_{0}$ can be calculated (using $\mathrm{t}_{\mathrm{c}}=2 \times 10^{-12} \mathrm{~s}$ ), provided the KWW exponent $\beta$, written as $(1-\mathrm{n})$ in eq 2 , is known. The fit by Lutz et al., using their modified KWW function for the neat $d_{4}$ PEO data, yiel ded a value of $\beta=0.33$, which the authors noted was unusually small. ${ }^{1}$ Molecular dynamics simulations of neat PEO found the stretch exponent $\beta$ to range from 0.33 to 0.48 for the torsional and $\mathrm{C}-\mathrm{H}$ vector autocorrelation functions. ${ }^{26}$ Small values of $\beta$ for PEO when $\tau_{\text {seg }}$ is in the 
subnanoseconds range are at odds with the results of other spectroscopies for longer times. The closely related poly(propylene oxide) (PPO) has a larger $\beta$, ca. 0.5 , as determined by dielectric spectroscopy for $\tau_{\text {seg }}$ in the millisecond range $^{27}$ (see Appendix), and in general we expect $\beta$ to increase with decrease in $\tau_{\text {seg }}$ (since at shorter times and/or higher temperature, cooperativity must be weaker). Comparable values of $\beta \sim 0.5$ are found by dielectric spectroscopy on other flexible polymers, including PVAC ${ }^{28}$ and poly(vinyl methyl ether) (PVME). ${ }^{29}$ In the absence of a reliable value of $\beta$ for neat $P E O$ in the subnanosecond range, we admit all possibilities in the range from 1.0 to 0.33 . F or example, the calculated $\tau_{0}$ is shown (dotted line) in Figure 1 for $\beta=$ $0.50(n=0.50)$.

In our previous analyses of blend dynamics, the independent relaxation time of any component in the blend was assumed to be the same value as for that same component in the neat state. This assumption has received support from recent experimental data on blends. The J ohari-Goldstein $\beta$-relaxation time, $\tau_{\text {J G }}$, has been identified with $\tau_{0}$ for a variety of glass-formers. ${ }^{30}$ Moreover, $\tau_{\mathrm{J}}$ of neat polymers is unchanged or changed only slightly upon blending, ${ }^{31}$ examples including the blends poly(ethyl methacrylate)/poly(4-vinylphenol),32 PVME/poly(4-vinylphenol),33 PVME/PS, 29 and 1,4-polybutadiene with aliphatic oil. ${ }^{34}$ Thus, $\tau_{i, 0}$ in eq 4 is taken as $\tau_{0}$ for neat $\mathrm{d}_{4} \mathrm{PEO}$, and the most probable segmental relaxation time $\tau(\hat{n})$ of $d_{4} P E O$ in a blend is calculated as

$$
\tau(\hat{n})=\left[\mathrm{t}_{\mathrm{c}}{ }^{-\hat{n}} \tau_{0}\right]^{1 /(1-\hat{\mathrm{n}})}
$$

assuming the most probable n of the distribution were known. However, the deuterium NMR data cannot be used for an unambiguous determination of the distribution of $\mathrm{n}$ and its possible temperature dependence. Since PMMA has a larger $\mathrm{T}_{\mathrm{g}}$ and is thus relatively immobile, it enhances the intermolecular constraints on segmental motion of $d_{4} P E O$ in the blend. Within the context of the coupling model, this means that $\hat{n}$ is certainly larger than $n$ for neat $d_{4} P E O$. The difference will increase with increasing PMM A concentration. Furthermore, since the difference between the segmental relaxation times of PMMA and $\mathrm{d}_{4} \mathrm{PEO}$ increases with decreasing temperature, a concomitant increase of $\hat{n}$ is possible. The open triangles in Figure 1 are calculated from eq 5 using n̂ $=0.715$. The open squares and open circles are calculated in similar fashion, with $\hat{n}=0.75$ and 0.76 , respectively. It can been seen that the calculated points match reasonably well the observed $d_{4}$ PEO segmental relaxation times in the $30 \% \mathrm{~d}_{4} \mathrm{PEO}$ blend and in the $3 \%-10 \% \mathrm{~d}_{4} \mathrm{PEO}$ blends. Our intention herein is not to fit these data (which are themselves the result of a model-dependent fitting procedure), but rather to demonstrate that our description of blend dynamics can account for the observed behavior. Specifically, despite the large increase of the coupling parameter from 0.50 in neat PEO to n̂ values larger than 0.7 in blends with the higher $\mathrm{T}_{\mathrm{g}} \mathrm{PMMA}$, the observed $\mathrm{d}_{4} \mathrm{PEO}$ segmental dynamics in the $30 \% \mathrm{~d}_{4} \mathrm{PEO}$ blend becomes at most about 10 times slower. Moreover, even for a sizable increase of $\hat{n}$ from 0.715 to $0.76,35$ the calculated $d_{4} P E O$ segmental dynamics are nearly independent of composition from $3 \%$ to $30 \% d_{4}$ PEO, in agreement with the experimental observations.

We believe it is unlikely that the coupling parameter of neat PEO can be much smaller than the value of 0.50

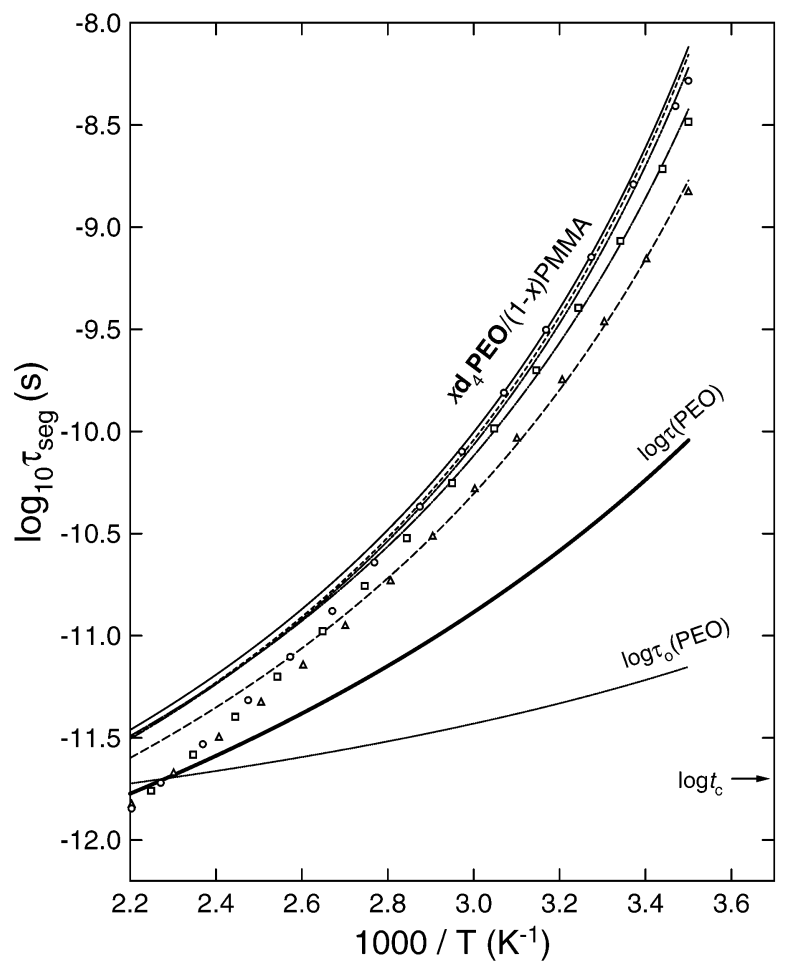

Figure 2. Segmental relaxation times for PEO neat (bold solid line) and in blends with PMMA containing 3\% (solid line) to $30 \%$ (dashed lines) PEO, as reported by Lutz et al. ${ }^{1}$ Shown by the dotted line is the independent relaxation time for neat PEO calculated using $n=0.67$, the value reported by Lutz et al. ${ }^{1}$ Also shown are the most probable relaxation times cal culated from eq 5 for the lower $(O, \hat{n}=0.84)$, midrange $(\square, \hat{n}=0.83)$, and higher concentrations of PEO $(\Delta, \hat{\mathrm{n}}=0.81)$.

of neat PPO, as determined by dielectric measurements (Appendix). Neverthel ess, we carried out similar calculations as above for the $d_{4} P E O$ component dynamics, but assuming $\hat{n}$ to be as low as $0.40(\beta=0.60)$ and as high as $0.67(\beta=0.33)$. In Figure 2 we illustrate the latter case, corresponding to the value of $\beta$ reported by Lutz et al. ${ }^{1}$ Note that the primary features are reproduced. Indeed, for any value assumed for $\beta$ in this range, we find that (i) an increase of the coupling parameter of the $d_{4}$ PEO component, due to immersion in the relatively rigid PMMA environment, accounts for the (small) increase of the segmental relaxation time, $\tau(\hat{n})$, and (ii) the calculated $\tau(\hat{n})$ are nearly independent of composition from $3 \%$ to $30 \% \mathrm{~d}_{4} \mathrm{PE}$, despite the increase of n̂ with decreasing $d_{4} P E O$ concentration. These two features, which are independent of the value of the coupling parameter for neat PEO, have their origin in the small value of the relaxation times at the high temperatures of the $d_{4}$ PEO deuterium NMR measurements. This is discussed immediately below.

The unusual results for the $d_{4}$ PEO/PMMA blend are a consequence of eq 5 , as can be seen by rewriting it in the form

$$
\tau(\hat{n}) / \tau_{0}=\left[\tau_{0} / t_{c}\right]^{\hat{n} /(1-\hat{n})}
$$

The independent relaxation time $\tau_{0}$ in the temperature range of the $d_{4}$ PEO deuterium NMR measurements is very short, ranging from $10^{-12}$ to $2 \times 10^{-11} \mathrm{~s}$ (see Figure $1)$. Since $t_{c}=2 \times 10^{-12} \mathrm{~s},{ }^{15,16}$ the ratio $\left[\tau_{0} / t_{c}\right]$ is not large, and consequently $\left[\tau_{0} / t_{c}\right]^{\hat{n} /(1-\hat{n})}$, and hence $\tau(\hat{n})$, do not vary much within the range of $\hat{n}$ engendered by the range of PMMA concentration. In the extreme case, $\tau_{0} \approx \mathrm{t}_{c}, \tau(\hat{n})$ 
becomes independent of $\hat{n}$; that is, it would become independent of PMMA concentration. On the other hand, if $\tau_{0}$ were much longer, $\tau(\hat{n})$ would be much larger than that of neat PEO, and thus a large variation of $\tau(\hat{n})$ with composition would result. This latter scenario is exemplified by other polymer blends, as described below. In these other blends, neither the observed nor calculated component dynamics behaves like $d_{4} P E O$ in PMMA as in Figures 1 and 2 because the experiments are carried out under conditions that the segmental dynamics of the neat polymer are much slower than for $\mathrm{d}_{4}$ PEO in the deuterium NMR work.

\section{Blends Exhibiting More “Usual” Dynamics}

An example of the more common dynamics of the mobile component in a polymer blend is 1,4-polyisoprene (PI) mixed with poly(vinylethylene) (PVE). 5,6 Shown in Figure 1 are the two-dimensional deuterium NMR data of the segmental relaxation time of $\mathrm{PI}$ neat and in $75 \%$, $50 \%$, and $25 \% \mathrm{PI}$ blends. ${ }^{36}$ These results, given in Figure 1 by their Vogel-Fulcher-Tammann-Hesse fits, have previously been shown to be in agreement with dielectric and mechanical relaxation data. ${ }^{6}$ Dielectric relaxation spectra for neat $\mathrm{PI}$ in the range $10^{2} \leq \tau_{\text {seg }} \leq 10^{-5} \mathrm{~s}$ are well-described by the one-sided F ourier transform of the $\mathrm{KWW}$ function with $\mathrm{n}=0.47$. Hence, in the temperature range of the $\tau$ in Figure 1, the independent relaxation time $\tau_{0}$ of $\mathrm{PI}$ is calculated by eq 2 using this value of $\mathrm{n}$ and is also shown in Figure 1.

As pointed out above, from eq 6 the factor determining how rapidly the segmental relaxation time $\tau_{\text {i }}$ of the faster component changes with composition is the ratio of its $\tau_{0}$ (or $\tau_{\mathrm{i}, 0}$ in the blends) to $\mathrm{t}_{\mathrm{c}}$. It is obvious from Figure 1 that $\tau_{0}$ of $\mathrm{PI}$ is much longer than $\tau_{0}$ of $\mathrm{PEO}$, and thus its ratio is much larger. It is this difference which is responsible for the large change of PI dynamics upon addition of PVE, in contrast to the near independence of the $d_{4} P E O$ dynamics to composition in blends with PMMA. To demonstrate this more quantitatively, we calculated $\tau_{\mathrm{i}}(\hat{\mathrm{n}})$ of $\mathrm{PI}$ in PI/PVE blends, with n̂ taken to increase from its value, 0.47 , for neat $\mathrm{PI}$ to 0.60 in the $50 \% \mathrm{PI}$ blend and to 0.67 in the $25 \% \mathrm{PI}$ blend. Assuming these coupling parameters to be temperature independent, the calculated $\tau_{\text {i }}(\hat{n})$ are shown as filled squares $(\hat{n}=0.60)$ and filled inverted triangles $(\hat{n}=$ $0.67)$ in Figure 1 . The calculated values are in approximate agreement with the experimental $\tau_{\mathrm{i}}(\hat{n})$ for $50 \%$ and $25 \% \mathrm{PI}$ in PVE blends. The values of $\hat{n}$ used herein are even smaller than those used above in our calculation of the $\tau_{\mathrm{i}}(\hat{\mathrm{n}})$ for the $\mathrm{d}_{4} \mathrm{PEO} / \mathrm{PMMA}$ blends with $3 \%-30 \% \mathrm{~d}_{4} \mathrm{PEO}$. F or a larger value of $\hat{n}$, such as 0.715 as for $\mathrm{d}_{4} \mathrm{PEO}$ in the blend with 70\% PMMA, the calculated $\tau_{\mathrm{i}}(\hat{\mathrm{n}})$ for PI in a 25\% PI in PVE blend would be even larger. The contrast with the virtual independence of $\tau_{\mathrm{i}}(\hat{n})$ to composition for the $\mathrm{d}_{4}$ PEO/PMMA blends is clear. The trend of increase of $\hat{n}$ with further decrease in PI concentration is also anticipated by our model, from the strengthening of intermolecular constraints, and concomitant increase in n̂, due to the increasing presence of the less mobile PVE component. Values of $n_{i}$ larger than 0.67 would result from low (say $3 \%) \mathrm{PI}$ concentrations, and via eq $6, \tau_{\mathrm{i}}(\hat{n})$ would be much larger than that shown in Figure 1 for the $25 \% \mathrm{PI}$ blend.

While the PI/PVE data considered above is for relatively low temperatures, NMR measurements at high temperature have also been carried out for the PI/PVE blend. ${ }^{37}$ The measurement frequencies, from 15 to 126

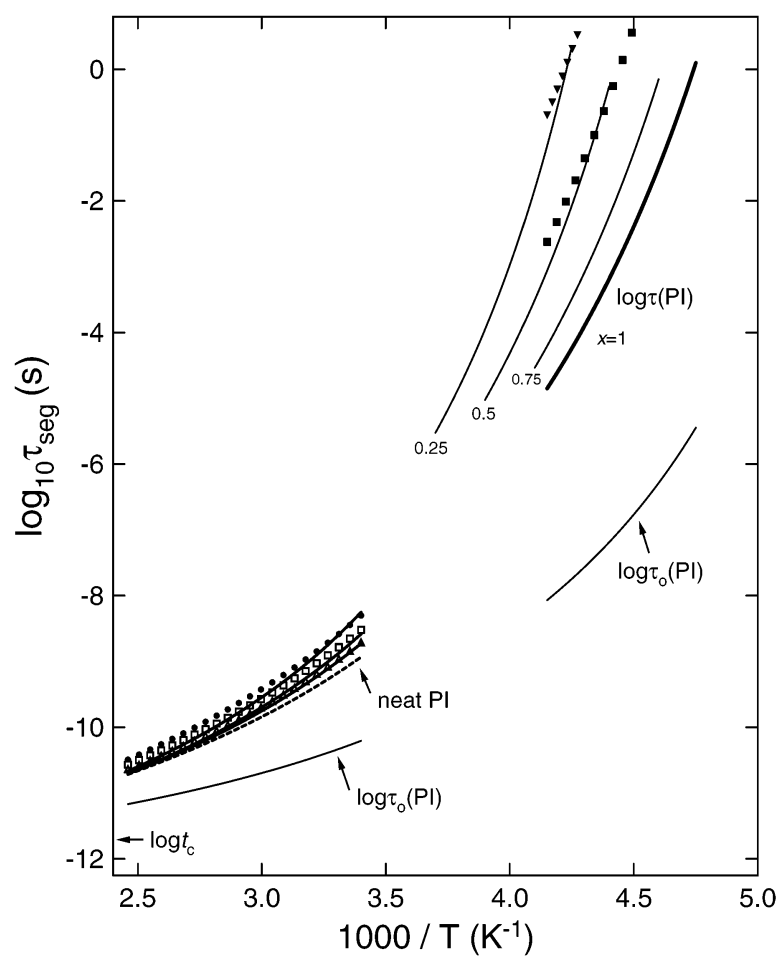

Figure 3. Data in lower left corner are the "average" segmental relaxation times for $\mathrm{PI}$ neat (dashed line) and in blends with PVE containing 70\%, 50\%, and 30\% PI (thicker solid lines, ascending order), as reported by Min et al. (ref 37). Shown by the lowest solid line is the independent relaxation time for neat $\mathrm{PI}$ calculated using $\mathrm{n}=0.46(\beta=0.54)$, the value reported by $\mathrm{Min}$ et al. ${ }^{37}$ Also shown are the most probable relaxation times calculated from eq 5 for the $70 \% \mathrm{PI}(\mathbf{\Lambda}, \hat{\mathrm{n}}=$ $0.54), 50 \% \mathrm{PI}(\square, \hat{n}=0.53)$, and $30 \% \mathrm{PI}(\bullet, \hat{\mathrm{n}}=0.56)$. The values of n used to calculate the most probable relaxation times correspond exactly to the values of $\beta(=1-\mathrm{n})$ for the blends given in Table 4 of ref 37.

$\mathrm{MHz}$, are comparable to the $31-76 \mathrm{MHz}$ range used in the study of $d_{4} P E O / P M M A$ blends. The same assumptions and procedure of ref 1 were used to deduce the segmental relaxation time, $\tau_{\text {seg, }}$ of $\mathrm{PI}$ and PVE and the KWW stretch exponents, $\beta$, of neat PI and PVE and their blends with 70,50, and 30\% PI. Since our interest is a comparison with the unusual d4PEO dynamics in the $d_{4}$ PEO/PMMA blends, we only consider the data for the fast PI component. The temperature dependences of $\tau_{\text {seg }}$ for neat $\mathrm{PI}$ and the three PI/PVE blends were presented ${ }^{37}$ in terms of the Vogel-Fulcher-TammannHesse (VFTH) equations; these are reproduced in Figure 3 (lower left corner). The dashed line is $\tau_{\text {seg }}$ for neat PI, and above that the thicker lines are the VFTH fits to the NMR data of the three blends (in ascending order with increasing PVE content). Comparing these data with $\tau_{\text {seg }}$ from the lower temperature N MR data ${ }^{36}$ (upper part of Figure 3), it is evident that the composition

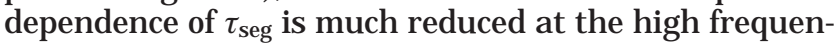
cies and higher temperatures of the measurements of Min et al. ${ }^{37}$ Substituting in eq 2 the "average" $\tau_{\text {seg }}$ given by Min et al. for $\tau$ and the "average" KWW exponent $\beta$ $=0.54$ of neat PI also from Min et al. (written as $1-\mathrm{n}$ in eq 2 ), the independent relaxation time $\tau_{0}$ is calculated (using $t_{c}=2 \times 10^{-12} \mathrm{~s}$ ) as a function of temperature. With the known $\tau_{0}$, we calculate the most probable segmental relaxation time $\tau(\hat{n})$ of $\mathrm{PI}$ in the PI/PVE blends by eq 5 with ( $1-\hat{n})$ exactly equal to $\beta$ given in Table 4 of $\mathrm{Min}$ et al.,37 i.e., $0.50,0.47$, and 0.44 respectively for the 70/30, 50/50, and 30/70 PI/PVE 
blends. Despite the absence of any adjustable parameters, the calculated $\tau(\hat{n})$ match the data well, although we emphasize again that our intention is not to fit these data (which themselves are the result of a modeldependent fitting procedure), but rather to demonstrate that our description of blend dynamics can account for the observed behavior, in particular, the reduced composition dependence of $\tau_{\text {seg }}$ at high frequencies.

On comparing the PI dynamics in the PI/PVE blends from Min et al. ${ }^{37}$ (Figure 3 ) with that of $d_{4} P E O$ in $d_{4}-$ PEO/PMMA blends ${ }^{1}$ (Figure 1 or 2), obviously the composition dependence of $\tau_{\text {seg }}$ is larger for the former ("usual" behavior) than for the latter. $\tau_{0}$ of $\mathrm{PI}$ is about an order of magnitude longer than $\tau_{0}$ of PEO, and it is this difference in $\tau_{0}$ that gives rise to the different composition dependences of $\tau_{\text {seg }}$ for the two blends.

Another miscible polymer blend cited by Lutz et al. ${ }^{1}$ as an example of "usual" component dynamics is PVME mixed with PS. Dielectric measurements of the PVME component dynamics were made by Pathmanathan et al. ${ }^{38}$ and by Zetsche et al. ${ }^{39}$ The latter results were shown ${ }^{4}$ to be in accord with eqs 3 and 5 of our model. A more comprehensive experimental investigation was subsequently undertaken by Cendoya et al. ${ }^{29}$ The segmental relaxation times of neat PVME and blends with $65 \%$ and $50 \%$ PVME were obtained by a combination of dielectric relaxation, ${ }^{13} \mathrm{C} N \mathrm{NM}$, and quasielastic neutron scattering experiments. These results are displayed in Figure 1 in the form of the fitted VogelFulcher-Tammann-Hesse curves. The data cover an enormous range of $\tau_{\text {seg, }}$ extending to times shorter than $10^{-10} \mathrm{~s}$. F or $10^{-5} \leq \tau_{\mathrm{seg}} \leq 10 \mathrm{~s}$, which is the same range as $\tau_{\text {seg }}$ for neat PI in Figure 1, substantial increases of $\tau_{\text {seg }}$ with decreasing PVME concentration can be noted. This large dependence of PVME's $\tau_{\text {seg }}$ on composition in this range follows directly from our model. To show this, we again calculate the relaxation times of PVME, using the literature value of $n=0.53$ for neat PVME, ${ }^{40}$ taken to be independent of temperature over the present range. Applying eq 5, with $n_{i}$ assuming a temperatureindependent value of 0.63 for $65 \%$ PVME in PS and 0.68 for $50 \%$ PVME in PS, we obtain the $\tau_{i}(\hat{n})$ displayed in Figure 1 . These fits can be improved by making $\hat{n}$ temperature dependent. However, our main purpose here is to show that values of nf for PVME/PS blends, which are even smaller than those for $\mathrm{d}_{4} \mathrm{PEO} / \mathrm{PMMA}$ blends, yield large changes of $\tau_{\mathrm{i}}(\hat{\mathrm{n}})$ in the former case. The calculations reproduce the experimental curves satisfactorily. Note that $\tau_{0}$ for PVME is not shown in Figure 1 since it would overlap the data points for PEO.

There is a new feature which can be observed in the PVME component dynamics, when $\tau_{\text {seg }}$ of neat PVME is small. As seen in Figure 1 , the dependence of $\tau_{\text {seg }}$ on composition becomes weaker as $\tau_{\text {seg }}$ of neat PVME decreases. Indeed, when $\tau_{\text {seg }}$ of PVME becomes comparable to $\tau_{\text {seg }}$ of $\mathrm{d}_{4} \mathrm{PEO}$, the former becomes similarly insensitive to composition. Thus, the nearly composition-independent dynamics of $d_{4}$ PEO in PMMA blends is not unique, being seen in PVME/PS under the right condition. The requirement is that $\tau_{0}$ be not much larger than $\mathrm{t}_{\mathrm{c}} \approx 2 \times 10^{-12} \mathrm{~s}$. Lutz et al. ${ }^{1}$ suggested that the unusual feature of $d_{4} \mathrm{PEO}$ might originate from its lack of a side group. However, the similarly behaving PVME is not lacking in a pendant group, having a methyl ether moiety, $-\mathrm{O}-\mathrm{CH}_{3}$, attached to its polyethylene backbone (although the difference in component $\mathrm{T}_{\mathrm{g}}$ 's for PVME/ PS is not as large as for PEO/PMMA).
Finally, we note that that most current models of miscible polymer blends focus primarily on the effect of concentration fluctuations to generate predictions. In the absence of concentration fluctuation, these models are not applicable. Thus, they offer no predictions concerning the component dynamics when the concentration becomes sufficiently dilute that each chain is surrounded by repeat units of the other component (notwithstanding chain connectivity, of course). The $0.5 \% \mathrm{~d}_{4} \mathrm{PEO}$ blend measured by Lutz et al. ${ }^{1}$ represents such a case, and the authors report the dynamics to be identical to that of 3,6 , and $10 \% \mathrm{~d}_{4}$ PEO compositions. Our model for the component dynamics, based on the coupling model, considers not only the presence of different local environments due to concentration fluctuations but al so the change of intermolecular coupling due to presence of the other component. Even at infinite dilution, without concentration fluctuations, the coupling parameter of the dilute component in a blend will differ from its value in the pure state. Our model can therefore yield predictions for the dynamics of that component.

An example of such an application is the dynamics of small probe molecules dispersed in various polymeric and nonpolymeric liquids. ${ }^{25}$ At infinite dilution, the binary blend is reduced to a neat polymer. Our coupling model based blend model remains applicable in this situation, having provided explanations for various viscoelastic anomalies found in amorphous polymers. ${ }^{11,14,41}$

Acknowledgment. The work was supported by the Office of Naval Research. We thank Mark Ediger and co-workers for hel pful comments.

\section{Appendix}

In the analysis of deuterium NMR measurements, the segmental relaxation function is not directly observed. The spin-relaxation times, obtained for a range of temperatures and different Lamor frequencies, are fit to a model in which the distribution function is a modified KWW function and the relaxation times have a VFTH temperature dependence. ${ }^{1}$ For isothermal dielectric spectroscopy measurements, the segmental relaxation function, $\phi(\mathrm{t})$, is obtained from the measured dielectric loss as

$$
\epsilon^{\prime \prime}(\omega)=\int_{0}^{\infty} \mathrm{dt}\left[\frac{-\mathrm{d}}{\mathrm{dt}} \phi(\mathrm{t})\right] \sin (\omega \mathrm{t})
$$

where $\omega$ is the angular frequency.

The lower frequencies of most dielectric instruments require lower temperatures for characterization of segmental relaxation, ${ }^{3-5,7}$ in comparison to the twodimensional deuterium NMR experiments. ${ }^{36}$ In the case of PEO, this precludes measurements on the amorphous state because of crystallization. However, di electric loss spectra of the chemically similar PPO were reported by Williams et al. ${ }^{42}$ The $\alpha$-loss peak, shown in Figure 4, is independent of temperature over the range studied. We fit the PPO data to eqs 1 and 7 , obtaining $n=0.5$. From this we can deduce an upper bound for the coupling parameter for PEO. We have previously shown ${ }^{9,10,13}$ that the magnitude of $\mathbf{n}$ (or the stretch exponent $\beta$ ) is related to chemical structure. Specifically, the latter engenders constraints on local motion, whose strength governs the breadth of the segmental relaxation function. Smooth, flexible chain backbones have smaller $\mathrm{n}$, while more 


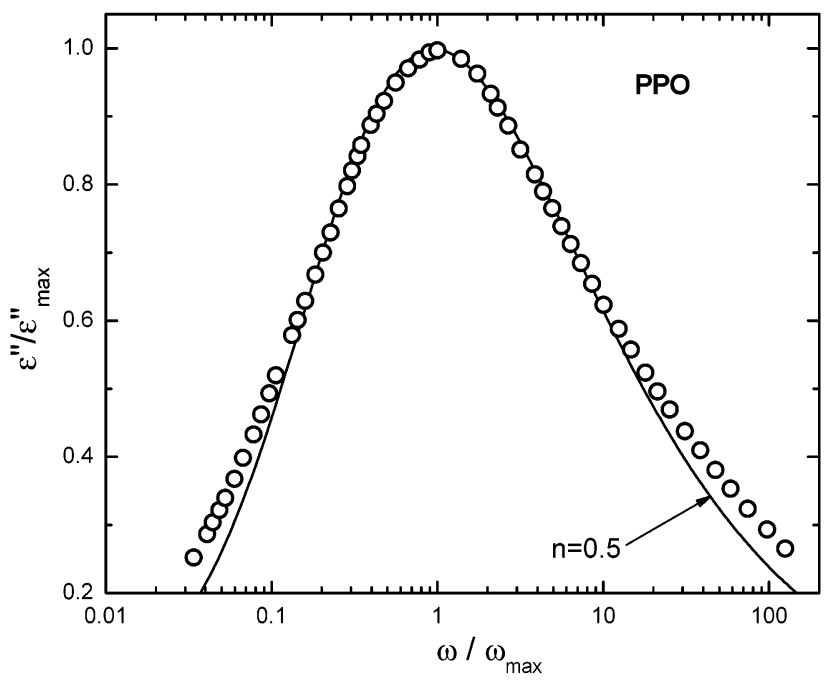

Figure 4. $\alpha$-Dispersion in the dielectric loss of poly(propylene oxide) from ref $42(\bigcirc)$, along with the fit to eqs 1 and 7 with $n$ $(=1-\beta)=0.5$.

rigid chains having bulky pendant groups are associated with larger coupling parameters. Comparing the structures of PPO and PEO, the latter is more flexible and lacks any pendant group (except $\mathrm{H}$ ); thus, $\mathrm{n}$ for $\mathrm{PEO}$ is unlikely to be larger than $\mathrm{n}$ for PPO and probably is smaller. (N ote that a similar line of reasoning explains the lower glass transition temperature of PEO.) Accordingly, for PEO $\mathrm{n} \leq 0.5$. The analyses in Figures 1 and 2 correspond to $n=0.5$ and 0.67 , respectively. Of course, a coupling parameter for PEO even smaller than 0.5 would strengthen our argument, although the insensitivity of $\tau_{\text {seg }}$ for PEO to composition does not depend much on $n$. $\tau_{0}$ remains close to $t_{c}$, independent of the magnitude of $n$.

\section{References and Notes}

(1) Lutz, T. R.; He, Y.; Ediger, M. D.; Cao, H.; Lin, G.; J ones, A. A. Macromolecules 2003, 36, 1724.

(2) Roland, C. M.; Ngai, K. L. Macromolecules 1991, 24, 2261.

(3) Roland, C. M.; N gai, K. L. J . Rheol. 1992, 36, 1691.

(4) Roland, C. M.; Ngai, K. L. Macromolecules 1992, 25, 363; 2000, 33, 3184.

(5) Alegria, A.; Colmenero, J .; Ngai, K. L.; Roland, C. M. Macromol ecules 1994, 27, 4486.

(6) Ngai, K. L.; Roland, C. M. Macromolecules 1995, 28, 4033.

(7) Roland, C. M.; Ngai, K. L.; O'Reilly, J. M.; Sedita, J. S. Macromol ecules 1992, 25, 3906.

(8) Ngai, K. L.; Tsang, K. Y. Phys. Rev. E 1999, 60, 4511. Ngai, K. L. IEEE Trans. Dielectr. Electr. Insul. 2001, 8, 329.

(9) Roland, C. M.; Ngai, K. L. Macromolecules 1991, 24, 5315; 1992, 25, 1844.

(10) Ngai K. L.; Roland, C. M. Macromolecules 1993, 26, 6824.

(11) Ngai, K. L.; Plazek, D. J .; Rendell, R. W. Rheol. Acta 1997, $36,307$.

(12) Ngai, K. L.; Roland, C. M. Macromolecules 1994, 27, 2454.
(13) Roland, C. M. Macromolecules 1994, 27, 4242.

(14) Ngai, K. L.; Plazek, D. J . Macromol ecules 2002, 35, 9136.

(15) Ngai, K. L.; Rendell, R. W. In Supercooled Liquids, Advances and Novel Applications; Fourkas, J. T., Kivelson, D., Mohanty, U., Nelson, K., Eds.; ACS Symposium Series Vol. 676; American Chemical Society: Washington, DC, 1997; Chapter $4, \mathrm{p} 45$.

(16) Colmenero, J .; Arbe, A.; Coddens, G.; Frick, B.; Mijangos C,; Reinecke, H. Phys. Rev. Lett. 1997, 78, 1928.

(17) Read, B. E. Polym. Eng. Sci. 1983, 23, 835. Osaki, K.; I noue, T.; Hwang, E.-J .; Okamoto, H.; Takiguchi, O. J . Non-Cryst. Solids 1994, 172-174, 838. Roland, C. M.; Mott, P. H. Macromolecules 1999, 32, 4728. Mott, P. H.; Roland, C. M. Macromol ecules 1998, 31, 7095. Roland, C. M.; Archer, L. A.; Mott, P. H.; Sanchez-Reyes, J. J . Rheol. 2004, 48, 395.

(18) Roland, C. M.; Santangelo, P. G.; Baram, Z.; Runt, J . Macromolecules 1994, 27, 5382.

(19) Santangelo, P. G.; Roland, C. M.; Ngai, K. L.; Rizos, A. K.; Katerinopoulos, H.J . Non-Cryst. Sol ids 1994, 172-174, 1084

(20) N gai, K. L.; Rizos, A. K. Macromolecules 1994, 27, 4493.

(21) Rizos, A. K.; Ngai, K. L. Phys. Rev. B 1992, 46, 8126.

(22) Roland, C. M. Macromolecules 1995, 28, 3463.

(23) Roland, C. M.; Santangelo, P. G.; Ngai, K. L.; Meier, G. Macromolecules 1993, 26, 6164.

(24) Rizos, A. K.; Ngai, K. L. Macromolecules 1994, 27, 7076.

(25) Ngai, K. L. J . Phys. Chem. B 1999, 103, 10684.

(26) Borodin, O.; Bedrov, D.; Smith, G. D. Macromol ecules 2001 34, 5687.

(27) León, C.; Ngai, K. L.; Roland, C. M. J . Chem. Phys. 1999 $110,11585$.

(28) Ngai, K. L.; Roland, C. M. Polymer 2002, 432, 567.

(29) Cendoya, I.; Alegria, A.; Alberdi, J . M.; Colmenero, J .; Grimm, H.; Richter, D.; Frick, B. Macromolecules 1999, 2, 4065.

(30) N gai, K. L. J . Phys.: Condens. Matter 2003, 15, S1107.

(31) The noncooperative relaxation time does not change upon blending if there is no significant chemical reaction between the components. Strong specific interactions, such as $\mathrm{H}$ bonding, could alter $\tau_{0}$.

(32) Zhang, S. H.; J in, X.; Painter, P. C.; Runt, J . Macromolecules 2002, 35, 3636.

(33) Zhang, S. H.; Painter, P. C.; Runt, J . Macromolecules 2002 , 35, 8478. Zhang, S. H.; Painter, P. C.; Runt, J . Macromolecules 2002, 35, 9403.

(34) Casalini, R.; Ngai, K. L.; Robertson, C. G.; Roland, C. M. J . Polym. Sci., Polym. Phys. Ed. 2000, 38, 1841.

(35) The dependence of $\tau(\hat{n})$ on n̂ is highly nonlinear (eq 5 ); thus, a change of n from 0.715 to 0.76 causes a very large increase in $\tau(\hat{n})$.

(36) Chung, G.-C.; Kornfield, J . A.; Smith, S. D. Macromolecules 1994, 27, 5729.

(37) Min, B.; Qiu, X.; Ediger, M. D.; Pitsikalis, M.; Hadjichristidis, N. Macromolecules 2001, 34, 4466. The "average" VFTH parameters, $\mathrm{B}, \mathrm{T}_{0}$, and $\tau_{\infty}$ given in their Table 4 , were used to reproduce $\tau_{\mathrm{seg}}$ of neat PI and the three blends with PVE in the lower left corner of Figure 3 herein.

(38) Pathmanathan, K.; J ohari, G. P.; Faivre, J . P.; Monnerie, L. J . Polym. Sci., Part B: Polym. Phys. Ed. 1986, 24, 1587.

(39) Zetsche, A.; Kremer, F.; J ung, W.; Schulze, H. Polymer 1990, 31, 1883.

(40) This value is obtained by fitting the KWW function to the data of neat PVME in ref 33.

(41) Plazek, D. J .; Chay, I.-C.; Ngai, K. L.; Roland, C. M. Macromolecules 1995, 28, 6432.

(42) Williams, G. Trans. Faraday Soc. 1965, 61, 1564.

MA035774P 